\title{
Spatiotemporal Patterns and Determinants of Undernutrition Among Late Adolescent Girls in Ethiopia Using Ethiopian Demographic and Health Surveys 2000 to 2016: Spatiotemporal and Multilevel Approach.
}

Nebiyu Derseh ( $\square$ nebiyumek12@gmail.com )

University Of Gondar

Kassahun Gelaye

University Of Gondar

Atalay Muluneh

University Of Gondar

Research Article

Keywords: Spatiotemporal patterns, Undernutrition, Late adolescent girls, Ethiopia

Posted Date: January 15th, 2021

DOl: https://doi.org/10.21203/rs.3.rs-141031/v1

License: (c) (1) This work is licensed under a Creative Commons Attribution 4.0 International License.

Read Full License 
1 Spatiotemporal Patterns and Determinants of Undernutrition among Late

2 Adolescent Girls in Ethiopia using Ethiopian Demographic and Health Surveys

32000 to 2016: Spatiotemporal and Multilevel approach.

4 Nebiyu Mekonnen Derseh ${ }^{1 *}$ Kassahun Alemu Gelaye $^{2}$ and Atalay Goshu Muluneh ${ }^{2}$

5 Authors affiliations:

$6{ }^{1}$ Department of Internal Medicine, school of Medicine, University of Gondar comprehensive

7 specialized Hospital, University of Gondar, Gondar, Ethiopia

$8 \quad 2$ Department of Epidemiology and Biostatistics, Institute of Public Health, College of Medicine

9 and Health Sciences, and Comprehensive Specialized Hospital, University of Gondar, Gondar,

10 Ethiopia

11 Contributor information:

12 *Corresponding Author: Nebiyu Mekonnen Derseh

13 Email: nebiyumek12@gmail.com, 


\section{Abstract}

2 Introduction: Under-nutrition of late adolescent girls in Ethiopia is the highest among Southern

3 and Eastern African countries. However, the spatial and temporal variations of under-nutrition as

4 a national context is not well understood. This study aimed the spatiotemporal patterns and

5 determinants of under-nutrition among Late Adolescent Girls in Ethiopia.

6 Methods: An in-depth secondary data analysis was conducted from women's data of four

7 consecutive Ethiopian Demographic and Health Surveys (EDHS) 2000 to 2016. A total of 12,056

8 late adolescent girls were included in this study. The global spatial autocorrelation was assessed

9 using the Global Moran's I statistic to evaluate the presence of geographical clustering and

10 variability of undernutrition. The significant cluster scan statistics using Bernoulli model to detect

11 local clusters of significant high rate and low rates of under-nutrition was explored. Multilevel

12 binary logistic regression model with cluster level random effects was fitted to determine factors

13 associated with under-nutrition among Late Adolescent girls in Ethiopia.

14 Results: undernutrition was clustered nationally during each survey (Global Moran's I=0.009-

$150.045, \mathrm{Z}$-score $=5.55-27.24$, $\mathrm{p}$ value $<0.001)$. In the final model, individual and community level

16 factors accounted about $31.67 \%$ of the variations for under-nutrition. The odds of being under-

17 nourished girls in the age groups of $18-19$ years were $57 \%$ (AOR $=0.43 ; 95 \% \mathrm{CI}: 0.35-0.53$ )

18 less likely than those from 15-17 years old. Being in higher educational status was 4.50 times

$19 \quad(\mathrm{AOR}=4.50 ; 95 \% \mathrm{CI}: 2.33-8.69)$ more likely to be under-nourished compared with no educational status. Undernutrition with occupation of sales was $40 \%$ (AOR=0.60; 95\% CI: $0.43-0.84$ ) lower than those with not working. The odds of being undernourished adolescents were 1.77 times

$22(\mathrm{AOR}=1.77 ; 95 \% \mathrm{CI}: 1.24-2.53)$ higher than participants with unimproved latrine type. Rural residents were 2. 35 times ( $\mathrm{AOR}=2.35 ; 95 \% \mathrm{CI}: 1.41$ - 3.92) more likely to be under nourish 24 compared with urban residents.

25 Conclusion: undernutrition among late adolescent girls was spatially clustered in Ethiopia. The 26 significant high rate of undernutrition was observed in Northern and Eastern Ethiopia. Those 27 regions with high rates of under-nutrition should design interventions to combat under-nutrition.

28 Key words: Spatiotemporal patterns, Undernutrition, Late adolescent girls, Ethiopia 


\section{Introduction}

2 Adolescence is the period of transition between childhood and adulthood (1) and late adolescent

3 girls is defined as those female adolescents aged 15-19 years (2). Adolescent girls are vulnerable

4 to undernutrition because of increased demand of nutrition for growth and development including

5 sexual development, maturation, and the onset of menarche (3). Adolescent females aged 15-19

6 years are more affected than women aged 20-49 years for under-nutrition in developing and middle

7 income countries (2). Under-nutrition among late adolescent girls is the major neglected public

8 health problem in developing countries including Ethiopia. South Asian region was the highest

9 burden for undernutrition among adolescent girls in the world (2), followed by Eastern Asian

10 region (2).

11 Sub-Saharan Africa was also the affected region with the burden of under-nutrition among late

12 adolescents girls (2). Ethiopia is the second populous country in Africa, next to Nigeria with an

13 increasing burden of household food insecurity that was affected by recurrent drought and land

14 degradation, population pressure, instability and social conflict that is liked with adolescent

15 undernutrition (4). Ethiopia was the first among Eastern and Southern African countries for late

16 adolescent girls under-nutrition (5). Under-nutrition among adolescent girls in Ethiopia is higher

17 compared with other African countries and continued to be the major public health problem (6).

18 During 2016 the prevalence of under-nutrition among late adolescence girls in Ethiopia was 29\%,

19 whereas the proportion of overweight or obese was low (3\%) (7). A local study conducted in

20 Northwestern Ethiopia remarked that nearly 50\% of adolescent girls were chronically

21 malnourished (8).

22 Under-nutrition results in many public health problems. It can affect educational attainment,

23 future productivity, and increased likelihood of infections like TB, pneumonia and even early

24 death (9). Evidence showed that undernourished adolescent girls are mostly from stunted young

25 and preschool children and they will continued to become malnourished mothers in the future who

26 in turn give birth of low birth weight babies which continues to become intergeneration cycle (10,

27 11). One in three (33\%) late adolescent girl gives birth during her adolescence period that is more

28 likely to be stunted compared to babies born to mothers who are older than 19 years of age (9). 
1 The impact of undernutrition also leads to stillbirths, small-for-gestational-age neonates,

2 complicated delivery and even maternal death among married late adolescent girls (7).

3 Undernourished adolescent girls are often highly vulnerable to multiple micronutrient deficiencies

4 such as iron deficiency anemia, Iodine deficiency disorder, Vitamin D and Vitamin A deficiencies;

5 all of which contribute to the risk of infectious and chronic diseases and leads to DALYs and

6 premature deaths $(3,12-14)$. A study done in rural Ethiopia revealed that $27 \%$ of undernourished

7 adolescent girls were anemic and late adolescents were four times higher than early adolescent for

8 developing anemia (15).

9 Therefore, an intervention of adolescent girls' under-nutrition contributes not only improving their quality of lives, but also a key target for $\mathrm{MCH}$ improvement. It reduces under five children stunting by $40 \%$, maternal anemia by $50 \%$, LBW by $30 \%$ globally (9).

12 Evidence showed that the causes of malnutrition are diverse and complex, but they are categorized as in to two dimensions: immediate causes like inadequate diet and diseases; underlying causes such as food insecurity, limited knowledge, local taboos, culture, inadequate health care access and poor hygiene-sanitation practices (10). Scholars also reported that age groups, being rural residence, having family size $\geq 5$, parents' educational status, dietary diversity score, absence of latrine in $\mathrm{HH}$, unprotected water source for drinking and food insecure households were determinant factors of under-nutrition among late adolescent girls $(6,8,16,17)$.

In Ethiopia, despite the increased health care coverage, and the government's good commitment towards agricultural production and productive safety net programs, under-nutrition among adolescent girls is the continued the major public health problem (5-7). A spatio-temporal study is useful for exploring high burden of under-nutrition overtime in specific geographical areas within a community in order to design community-based interventions in such areas. Therefore, understanding the spatio-temporal patterns and determinates of under-nutrition among late adolescent girls are important for evidence-based decision making to improve adolescents, women and also future childhood nutritional status in Ethiopia. However, the spatio-temporal distributions and determinants of under-nutrition among late adolescent girls at national context is not well understood in Ethiopia. Therefore, this study contributed spatio-temporal patterns and associated factors of under-nutrition among late adolescent girls in Ethiopia that is important to design better interventions at community levels in the country. 


\section{Methods}

\section{The study settings}

3 This was national level study represented 11 regions of urban and rural areas of Ethiopia from data

4 collected Ethiopia Demographic and Health Surveys (EDHS) 2000 to 2016. EDHS is the

5 comprehensive and nationally representative surveys conducted in Ethiopia since 2000 as a part

6 of worldwide DHS program every five years interval (7). Ethiopia is located in the horn of African

7 and lies between latitudes between $3^{\circ}$ and $15^{\circ}$ North, and longitudes between $33^{\circ}$ and $48^{\circ}$ East. It

8 has a total area of 1,100,000 km2. There are nine ethnic based and politically autonomous regional

9 states and two administrative cities (Addis Ababa and Dire-Dawa) in Ethiopia. The Regions are

10 subdivided into sixty-eight zones, and then further into 817 districts which are further divided into

11 around 16,253 Kebels, the lowest local administrative units (7). Ethiopia is a country with great

12 geographical diversity; its topographic features range from the highest peak at Ras Dashen, which

13 is 4,550 meters above sea level, down to the Afar Depression at 110 meters below sea level. The

14 climatic condition of the country varies with the topography and temperatures as high as $47^{\circ} \mathrm{c}$ in

15 the Afar Depression and as low as $10^{\circ} \mathrm{c}$ in the highlands. Ethiopia is an agrarian country, and the

16 agriculture is the backbone of the national economy (18). Ethiopian population growth is fast (53.5

17 million in 1994 census increased to be 114,963,588 in 2020 and under 25 years are 60\% (19).

Study design and period

This was community based nationally representative cross-sectional survey study in four consecutive time periods from 2000 to 2016.

\section{Population and Eligibility criteria}

22 The source population for this study were all non-pregnant and non-postpartum adolescent girls 23 aged 15 to 19 years preceding 5 years before the survey in Ethiopia, while the study population were all non-pregnant and non-postpartum adolescent girls aged 15 to 19 years in Ethiopia who were in the selected EAs and included in the analysis. Adolescent girls whose outcome variable was not measured were excluded from the study, but their characteristics had no major difference

27 from others. 
2 We accessed the datasets using the website www.measuredhs.com after requested from the DHS

3 program database. A total of 12, 056 late adolescent girls (3, 545 in 2000, 1,550 in 2005, 3,700 in

42011 and 3, 261 in 2016) were included in this study. Sampling weight was done to make

5 representativeness in each survey. EDHS sample was a representative sample of randomly selected

6 from the target population. Each interviewed unit (household and individual) represented a certain

7 number of similar units in the target population (20). A two-stage stratified cluster sampling

8 techniques for each EDHS data was used and details of methodology was presented from each

9 EDHS report. In the first stage; a stratified sample of census enumeration areas (EAs) in the urban

10 and rural areas were selected with complete household listing using systematic probability

11 sampling based on sampling frame with population and household information from the 1994 and

122007 PHC. In the second-stage: the selection of households was carried out by equal probability

13 systematic sampling in the selected EAs. In each selected household, late adolescents were

14 interviewed with an individual questionnaire $(5,7,21,22)$.

\section{Study Variables}

16 The outcome variable: was late adolescent girls' under-nutrition. It was measured with body

17 mass index less than $18.5 \mathrm{~kg} / \mathrm{m}^{2}$ which was recorded as binary outcome (1- for under-nourished

$18\left(\mathrm{BMI}<18.5 \mathrm{BMI} \mathrm{kg} / \mathrm{m}^{2)}\right.$ and else was recorded as 0$)$.

19 Independent variables: individual level factors (level one) like Scio-Demographic and Socio-

20 Economic characteristics and community level factors (level two), are considered to be

21 determinants of under-nutrition.

\section{Individual level factors (level one):}

23 Socio-demographic factors: Age of adolescent girls, educational status of adolescents, literacy, 24 marital status of adolescents, occupation of adolescents, media exposure, religion, head of $\mathrm{HH}$ sex 25 and age, number of $\mathrm{HH}$ members, number of $<5$ children.

26 Socio-economic characteristics: Wealth index, source of drinking water, time to get water, type 27 of latrine, toilet facilities shared with other $\mathrm{HH}$, anemia status, type of cooking fuel, khat chewing, 28 alcohol drinking, covered by health insurance 


\section{Community level factors (level Two):}

Region, residence, un-protected water source in the community, community poverty status, community adolescent's educational achievement, unimproved latrine type in the community.

\section{Operational Definitions}

Under-nutrition: is defined as nutritional status for which Body Mass Index is less than 18.5 $\mathrm{kg} / \mathrm{m}^{2}$ which is either stunting or underweight (thinness) (23)

Stunting: Is chronic malnutrition status that is measured by height-for-age z-score of less than 2SD of the World Health Organization or height $<145 \mathrm{~cm}$ (2).

Total underweight (thinness): is defined as both acute and chronic malnutrition status that is measured by BMI-for-age z-score less than -2SDs from median or BMI $<18.5$ $\mathrm{kg} / \mathrm{m}^{2}(2)$.

Normal: is defined as well-nourished individuals with BMI-for-age z-score -2SD to $+1 \mathrm{SD}$ or BMI $18.5 \mathrm{~kg} / \mathrm{m} 2$ to $24.9 \mathrm{~kg} / \mathrm{m}^{2}$ (2).

Wealth index: A composite measure of a household's cumulative living standard divided into 5 quintiles using the wealth quintiles data, are derived using principal component analysis (7).

Community adolescent girls' educational achievement: A composite measure of adolescent girls' educational achievement in the community measured with aggregation of primary, secondary and higher educations categorized in to (1) low [0-0.75] (2) moderate [0.75-0.99] (3) high [1] divided in to 3 quantiles (7).

Community un-protected water source of drinking: It is the proportions of un-protected dug well and spring, tanker truck and surface water in the community measured above and below median value which was categorized in to (1) not big problem [0-0.125] (2) big problem [0.125-1] (7).

\section{Data collection and tools}

EDHS data was collected through face-to-face interviews using questionnaires at the individual and house-hold levels. During each EDHS data collection period, women aged 15 to 19 were asked to give important sociodemographic and economic status and maternal characteristics related to adolescent under-nutrition in chronological order before five years of each survey $(5,7$, $21,22)$. 
1 Data management and analysis

2 After extraction, the data were further cleaned, recoded, labeled for further analysis using STATA

3 version 14 and Microsoft excel. Sampling weight of each variable was done before conducting

4 analysis to restore for the unequal probability of selection between the strata.

5 Spatial Analysis: The global spatial autocorrelation was assessed using the Global Moran's I

6 statistic (Moran's I) to evaluate the presence of geographical clustering and variability using

7 ArcGIS Version.10.6. A positive value for Moran's Index indicated a geographical clustering for

8 undernutrition, while a negative value for Moran's Index indicated a dispersion and if zero value

9 distributed randomly, when Moran's Index is statistically significant. The local Getis-Ord spatial

10 statistical tool was used to identify statically significant hotspot and cold spot areas. Hotspot refers

11 to the occurrence of high prevalence of under-nutrition clustered together on the map, whereas

12 cold spots refers to the occurrence of low prevalence of undernutrition clustered together on the

13 map.

14 Kriging interpolation method was used to predict the spatial distribution of undernutrition from unsampled areas in Ethiopia.

We explored spatio-temporal scan statistics using Bernoulli probability model to detect local clusters of significant high rate and low rates of undernutrition using SaTScan 9.6 software. A cluster is reported to be statistically significant when its log likelihood ratio (LLR) is greater than the Standard Monte Carlo critical value (C.V) for p-value $<0.05$. The maximum likelihood ratio test statistic showed the most primary cluster compared with the overall distribution of maximum values. The primary and secondary clusters were identified and LLR was assigned and the p-value was obtained through the Monte Carlo hypothesis testing with 999 Monte Carlo replicates.

Multilevel analysis: Multilevel binary logistic regression model was fitted to identify the possible factors associated with under-nutrition among late adolescent girls in Ethiopia using STATA 14. We considered to use multilevel models, because each interviewed unit (household and individual) are hierarchical and nested to EAs (7). Therefore, a two-level model was adopted, by taking secondary sampling unit (individuals and households) as level-1 units and primary sampling units (EAs) as level-2 units. The multilevel binary logistic regression model incorporates fixed 
1 data. Therefore, the two-level fixed and random-effect logistic regression model was presented as

2 follow:

$3 \quad \operatorname{Logit}(Y i j)=\beta_{0 j}+\sum \beta X i+\Upsilon Z j+\varepsilon j$

$4 \quad \beta_{0 j}=\beta_{0}+\mu \mathrm{j}, \mu \mathrm{j} \sim \mathrm{N}(0, \sigma 2 \mathrm{u})$

$5 \varepsilon j=\varepsilon_{0}+\varepsilon j, \varepsilon j \sim N\left(0, \sigma^{2} \varepsilon\right)$

6 In this model, logit $(Y i j)=\ln (Y i j /(1-Y i j))$ is log-odds for undernutrition called 'the logit link'.

7 The symbol, 'Yij' is a probability of under-nutrition for an adolescent girl $\mathrm{i}$ in any EA, rural/urban

8 region, ' $\mathrm{j}$ '.' $\beta_{0 j}$ ' is the cluster random intercept. ' $\varepsilon j$ ' is the residual for each cluster ' $\mathrm{j}$ '. ' $\beta$ ' is fixed

9 effect regression coefficients and 'Xi's are level-1 predictors and $Y Z j$ are level-2 factors

10 (community level) in cluster $\mathrm{j}$.

11 We considered four models to be fitted for multilevel analysis:

12 Model I: Empty model which has no any individual or community level variables; Model II:

13 adjusted for individual-level variables, Model III: adjusted for the community level variables and

14 Model IV: adjusted for both the individual and community level variables.

15 Model comparison was done using Akaike's Information Criterion (AIC) and the model with the smaller value of AIC was selected as the final best fitted model.

17 Adjusted Odds Ratios (AOR) with their corresponding 95\% CI was calculated to identify the 18 determinants of under-nutrition with P-value of $<0.05$.

19 In random effects model, we computed Intra-class Correlation Coefficient (ICC), Median Odds Ratio (MOR) and Proportional Change in Variance (PCV) statistics for measures of variation

21 between clusters.

22 The fixed effects model has only one source of variability ( $\varepsilon j$, with its variance $\sigma^{2} \mu$ ), while the random effects model has two components of variabilities ( $\varepsilon j$ and $\varepsilon_{0}$ with variances $\sigma^{2} \mu$ and $\sigma^{2} \varepsilon$ respectively). These two sources of variability showed the variability between predictors that are 25 in the same group, measured by the within-group variance $\sigma^{2} \mu$, and the variability between observations that are in different groups, measured by the between-group variance $\sigma^{2} \varepsilon$. The 27 proportion of between-group variance $\left(\sigma^{2} \varepsilon\right)$ to total variance $\left(\sigma^{2} \mu+\sigma^{2} \varepsilon\right)$ is called intra-class 28 correlation (ICC) (25). It is calculated using the formula: 


$$
\operatorname{ICC}(\rho)=\frac{\sigma^{2} \varepsilon}{\sigma^{2} \varepsilon+\sigma^{2} \mu}
$$

$$
\sigma^{2} \mu=\frac{\pi^{2}}{3}=3.29 \text {--------- within-group variance }\left(\sigma^{2} \mu\right)
$$

3 The ICC quantifies the variation of the under-nutrition within clusters. The ICC may range from

40 to 1 . ICC $=0$ showed perfect independence of residuals and the observations do not depend on

5 clusters. However, ICC $=1$ or less than one indicates interdependence of residuals i.e. the variation

6 of observations between clusters (26).

7 The MOR (Median Odds Ratio) is defined as the median value of the odds ratio between the area

8 at highest risk and the area at lowest risk when comparing two individuals from two different

9 randomly selected clusters and it measures the unexplained cluster heterogeneity. The variation is between clusters by comparing two persons from two randomly chosen different clusters. It is

11 calculated as the following formula:

$12 \quad$ MOR $=\exp \sqrt{2} x V A^{2} x 0.6745=\exp (0.95 \times \mathrm{VA})$

$13 \mathrm{VA}$ is the estimated variance of clusters. The MOR is always greater than or equal to 1. If the 14 MOR is 1 , there is no variation between clusters.

15 The total variation attributed to individual and cluster level factors at each model was measured 16 by the proportional change in variance (PCV), which is computed as:

$17 \quad \mathrm{PCV}=\frac{V A-V B}{V A} \times \mathbf{1 0 0}$

18 VA is variance of the initial model and VB variance of the model with more terms

\section{Results}

\section{Socio-Demographic and Economic characteristics of participants}

During EDHS 2016, more than half 1,949 (60\%) of late adolescent girls were from the age group of 15-17 years and their median age and its IQR were 17 and 2.00 respectively. The majority of the study participants 2,513 (78\%) were never married. The majority 2,569 (80\%) of them were

24 from household of un-improved latrine type. Nearly 1,022 (32\%) of households had unprotected drinking water source. Among participants 455 (14.12\%) and $536(16.63 \%)$ were the poorest and the poorer respectively (Table 1). 
Table 1: Socio-demographic and economic characteristics of respondents included in the EDHS 2016 analysis.

\begin{tabular}{|c|c|c|c|}
\hline \multicolumn{2}{|c|}{ Socio-Demographic and Economic characteristics } & \multirow{2}{*}{$\begin{array}{l}\text { Weighted frequency } \\
1,948.94\end{array}$} & \multirow{2}{*}{$\begin{array}{l}\text { Percent } \\
60.5\end{array}$} \\
\hline Age groups: & $15-17$ & & \\
\hline & $18-19$ & $1,273.42$ & 39.5 \\
\hline \multirow[t]{5}{*}{ Religion: } & Orthodox & $1,374.31$ & 42.65 \\
\hline & Catholic & 20.23 & 0.63 \\
\hline & Protestant & 793.01 & 24.61 \\
\hline & Muslim & $1,013.12$ & 31.44 \\
\hline & Others & 21.69 & 0.67 \\
\hline \multicolumn{2}{|c|}{ Current marital status: never married } & $2,513.15$ & 77.99 \\
\hline & Married & 545.59 & 16.93 \\
\hline & living with partner & 19.12 & 0.59 \\
\hline & Widowed & 1 & 0.03 \\
\hline & Divorced & 96.31 & 2.99 \\
\hline & Separated & 20 & 0.61 \\
\hline \multicolumn{2}{|c|}{ source of drinking water: protected } & $2,084.92$ & 64.70 \\
\hline & un-protected & $1,021.77$ & 31.71 \\
\hline & Others & 115.67 & 3.59 \\
\hline \multicolumn{2}{|c|}{ Time get drinking water: Less than 30 minutes } & 988.07 & 30.66 \\
\hline & 30 minutes or longer & $1,366.99$ & |42.42 \\
\hline & Water on premises & 742.71 & 23.05 \\
\hline & Others & 124.58 & 3.87 \\
\hline \multirow[t]{3}{*}{ Type of toilet facility: } & Improved & 533.53 & 16.56 \\
\hline & Not improved & $2,568.62$ & 79.71 \\
\hline & Others & 120.21 & 3.73 \\
\hline \multirow[t]{5}{*}{ Wealth index } & poorest & 454.99 & 14.12 \\
\hline & Poorer & 535.94 & 16.63 \\
\hline & Middle & 608.87 & 18.90 \\
\hline & Richer & 687.32 & 21.33 \\
\hline & Richest & 935.23 & 29.02 \\
\hline
\end{tabular}

3 Community level characteristics of late adolescent girls' under-nutrition in Ethiopia

4 Among under-nourished late adolescent girls, the majority (83.89\%) of them were living in rural

5 areas of Ethiopia (Table 2). This study showed that the prevalence of under-nutrition among late

6 adolescent girls had decreased over time for the past 15 years. It had decreased from 36.79\% (95\%

7 CI: 34.11-39.54) in EDHS 2000 to 28.22\%, 95\% (CI: 26.02-30.52\%) in EDHS 2016, but it was 
1 increased from 30.86 (95\% CI: $27.84-34.04)$ in 2005 to $34.82 \%$ (95\% CI: $32.27-37.47$ ) in

2 2011(Table 3).

3 Table 2: Community level characteristics of respondents included in the EDHS 2016.

\begin{tabular}{llll}
\hline $\begin{array}{l}\text { Community } \\
\text { variables }\end{array}$ & $\begin{array}{l}\text { not under-nourished } \\
\text { Weighted Freq. }(\%)\end{array}$ & $\begin{array}{l}\text { under-nourished } \\
\text { Weighted Freq. }(\%)\end{array}$ & Total (\%) \\
\hline Residence: Urban & $604.049(80.49)$ & $146.45(19.51)$ & $750.50(100)$ \\
$\quad$ Rural & $1,709.06(69.14)$ & $762.80(30.86)$ & $2,471.86(100)$ \\
Community poverty & & & \\
status: Lower & $1,234.12(70.74)$ & $510.56(29.26)$ & $1,744.67(100)$ \\
$\quad$ Higher & $1,078.99(73.02)$ & $398.69(26.98)$ & $1,477.68(100)$ \\
Community un-improved latrine & & $1,026.37(100)$ \\
not big problem & $747.76(72.85)$ & $278.61(27.15)$ & $2,195.99(100$ \\
big problem & $1,565.35(71.28)$ & $630.64(28.72)$ &
\end{tabular}

Table 3: The prevalence of under-nutrition among late adolescent girls in Ethiopia, EDHS 2000, $5 \quad 2005,2011$ and 2016

\begin{tabular}{llll}
\hline Sr.No. & EDHS & Weighted Prevalence of under-nutrition $\%$ & $\mathbf{9 5 \%} \mathbf{C I}$ \\
\hline $\mathbf{1}$ & 2000 & 36.79 & $34.11-39.54$ \\
$\mathbf{2}$ & 2005 & 30.86 & $27.84-34.04$ \\
$\mathbf{3}$ & 2011 & 34.82 & $32.27-37.47$ \\
$\mathbf{4}$ & 2016 & 28.22 & $26.02-30.52$ \\
\hline
\end{tabular}

There was temporal and regional variation of under-nutrition among late adolescent girls in Ethiopia. In EDHS 2000 the prevalence of under-nutrition was higher (greater than national average) in regions of Afar (38.93\%), SNNPR (40.94\%), Tigray (42.55\%), Amhara (42.72\%), Ben-Gumuz (48.16\%), and Somali region (54.64\%), whereas in regions of Addis Ababa (23.28\%), Oromia (31.93\%), Harari (33.43\%) and Dre-Dawa (34.95\%) was lower. Similarly, in EDHS 2005 the prevalence of under-nutrition was higher in Somali (57.39\%), Tigray (46.43\%), Afar (46.34\%), Amhara (37.87\%) and Dre-Dawa (37.50\%) regions, while Addis Ababa (18.15\%), Harari (21.95\%), Oromia (25.43\%) and SNNPR (27.13\%) were observed as lower. Likewise, in 2011, the under-nutrition among late adolescent girls was reported as higher in regions of Tigray (52.31\%), Afar (46.74\%), Amhara (44.86\%) and Somali (42.48\%), while Addis Ababa (21.25\%), SNNPR (24.33\%), Gambela (26.08) and Dire-Dawa (29.22\%) were reported as lower. During 
EDHS 2016, the higher prevalence of under-nutrition was observed in the regions of Somali

2 (45.14\%), Tigray (43.31\%) and Afar (42.99\%), Gambela (38.29\%) Amhara (34.32\%), regions whereas SNNPR (17.37\%), Addis Ababa (20.35\% and Benishangul Gumuz (20.78\%) were reported as lower prevalence. Four regions (Tigray, Afar, Amhara and Somali) had higher 5 prevalence in 2000 to 2016 EDHS period, while Addis Ababa and Oromia were in lower 6 prevalence (Table 4).

7 Spatiotemporal distributions of under-nutrition among late Adolescent girls in 8 Ethiopia, EDHS 2000, 2005, 2011 and 2016

9 During EDHS 2000, the prevalence of under-nutrition among late adolescent girls was spatially distributed with regional variations. In the figure below, the red color showed the highest

11 prevalence of under-nutrition that was observed in Eastern \& Southern Tigray, eastern Amhara, 12 Somali region, southwestern Oromia region, SNN, while the green color was with the lowest prevalence which covered Tigray, Amhara, Oromia, SNNPR, Gambela regions (Fig. 1 A); Similarly, in EDHS 2005, the highest prevalence of under-nutrition was observed in central and southern Tigray, Amhara region eastern, SNNPR and Gambela region, while the lowest prevalence was distributed throughout the regions in the country (Fig. 1 B); In EDHS 2011, the highest prevalence was determined in regions of Tigray, Afar, Eastern Amhara, Gambela, whereas the low prevalence (blue color) was distributed throughout all of the regions, 19 (Fig.1 C);

Likewise, EDHS 2016 showed that the highest prevalence of under-nutrition among late adolescent girls was observed in Southern Tigray, Eastern Amhara, Gambela, Somali region and 22 Harari, while western Amhara region, Oromia region, Benishangul Gumuz, SNNPR, Addis Ababa, Dire-Dawa were in the lowest prevalence of under-nutrition (Fig. 1, D). Each point data on the map below represents one cluster that showed a number of under-nutrition 25 cases. The rate of under-nutrition was persistent over 15 years in regions Tigray, Afar, Northern \& Eastern Amhara, Southern Somali region and Gambela region.

27 Figure 1: Spatial distribution of late adolescent girls' under-nutrition in Ethiopia, EDHS 2000 to 282016. 
2 The spatiotemporal patterns of under-nutrition among late adolescent girls were not-random in

3 Ethiopia in each consecutive EDHS period. The global spatial autocorrelation analysis of each

4 survey showed that there were significant clustered patterns of under-nutrition across the country

5 (Global Moran's I=0.042, Z-score= 5.55, p value < 0.001 in EDHS 2000; Global Moran's I=0.009,

6 Z-score = 5.94, p value < 0.001 in EDHS 2005; Global Moran's I=0.045, Z-score =27.24, p value

$7<0.001$ in EDHS 2011 and Global Moran's $\mathrm{I}=0.030$, Z-score $=21.92$, p value $<0.001$ in EDHS

8 2016). Generally, in each output, the Z-score is high and positive with highly significant p-value

9 which showed $99 \%$ confidence for clustering of under-nutrition across regions in Ethiopia (Fig.2

10 A-D). The figures bellow showed that the clustered patterns (on the right side) of high rates of

11 under-nutrition across regions in Ethiopia. The bright red and blue colors (to the right and left

12 side) indicated increased significance level for which the likelihood of clustered patterns occurred

13 by random chance were less than $1 \%$ (Fig.2 A-D).

Figure 2: Spatial patterns of undernutrition among late adolescent girls in Ethiopia (2000, 2005, 2011 and 2016).

\section{Hot spot Analysis (Getis-Ord Gi*) of undernutrition among late adolescent girls in}

\section{Ethiopia, EDHS 2000 to 2016}

During EDHS 2000, the hot spot areas of under-nutrition among late adolescent girls were observed in regions of Tigray, Afar, Eastern Amhara, Southwest and South East Oromia, Somali, SNNPR, whereas western Amhara region and Northern and Eastern Oromia were reported as clod spots (Fig. 3 A). Similarly, in 2005, the hot spot areas of under-nutrition were observed in regions of Tigray, Afar, Northern and Eastern Amhara, while, the cold spot areas were seen in southern parts of Amhara region, Oromia region, Benishangul Gumuz, SNNPR and Addis Ababa (Fig.3 B). Likewise, in 2011, the hot spot areas of under-nutrition among late adolescent girls in Ethiopia were identified in Tigray, Western and Eastern Afar, Northern and Eastern Amhara, while the cold spot areas were seen Southern Amhara, Eastern Oromia, Southern Somali, and Benishangul Gumuz and Gambela regions (Fig. 3 C). During EDHS 2016, statistically significant hot spot areas were observed in regions of Tigray, Northern and eastern Amhara region, Afar, Eastern Somali region, and Gambela. The statistically significant cold spot areas were observed in regions 
of Southern Amhara, Oromia, SNNR, Benishangul Gumuz, Harari, Addis Ababa, Dire-Dawa (Fig.3 D). From the figure below, red and yellow colors showed significant clusters of high risk (hotspot) areas of undernutrition while green and blue colors showed significant clusters of low risk (cold spot areas)

Figure 3: Hotspot analysis of under-nutrition among late adolescent girls in Ethiopia, 2000, 2005, 2011 and 2016

\section{Cluster and Outlier Analysis (Anselin Local Moran's I) of undernutrition among late adolescent girls in Ethiopia, 2000, 2005, 2011 and 2016}

During EDHS 2000, statistically significant high-high local clusters (high rates of undernutrition) were observed in Tigray, southern Afar region, Eastern Amhara, Southwest and Southeast Oromia, Somali region, Northern SNNPR region whereas, the local low-low clusters were observed in Addis Ababa and around its borders, Southern Oromia, Gambela and Dire-Dawa. The significant outliers such as low-high were happened in Central Tigray, Eastern Amhara, Southwest Oromia, Somali region, SNNPR, while high-low outliers were observed in Eastern Amhara, Northern, Southern and Eastern Oromia and Benishangul Gumuz (Fig. 4 A).

Likewise, EDHS 2005 showed that the significant high-high clusters were observed in Tigray and Northern Amhara regions, while the low-low clusters were occurred in most parts of Oromia, Addis Ababa, SNNPR and Benishangul Gumuz regions. The low-high outlier was seen in Eastern and southern Tigray; Northern and Eastern Amhara and Eastern Afar; whereas high-low clusters were seen in East Amhara, Northern and Southwest Oromia, Northern and western SNNPR and Benishangul Gumuz (Fig. 4 B).

The local cluster and outlier analysis of EDHS 2011 revealed that the significant high-high significant clusters were identified in Tigray, Amhara (Northern and Eastern) and Afar, whereas the low-low clusters were occurred in Addis Ababa, Oromia, SNNPR, and Benishangul Gumuz regions. The low-high outliers were observed in Tigray, Afar and Eastern Amhara, while Addis Ababa, Oromia, SNNPR and Benishangul Gumuz were reported as high-low outliers. (Fig. 4 C). Similarly, EDHS 2016 showed that the high-high significant cluster areas were observed in regions of Tigray, Northern and Eastern Amhara, Afar, Eastern Somali and Gambela region, whereas the low-low significant clusters were observed in Southern Amhara, Addis Ababa, Dre-Dawa, 
Oromia, SNNPR and Benishangul Gumuz regions. The low-high outliers were observed in Tigray

2 region, Afar, Northern and Eastern Amhara and Gambela regions while Addis Ababa, Dire-Dawa,

3 Southern Amhara, Oromia region, SNNPR, Southern Somali and Benishangul Gumuz regions

4 were reported as high-low outliers (Fig. 4D). From the figure below, High-High means high rates

5 of undernutrition surrounded by high rates; High-Low means high rates of undernutrition

6 surrounded by low rates of undernutrition; Low-High means low rates of undernutrition

7 surrounded by high rates. Low-Low means low rates of undernutrition surrounded by low rates.

$8 \quad$ Figure 4: Cluster and Outlier Analysis of undernutrition among late adolescent girls in Ethiopia,

$9 \quad$ EDHS 2000 to 2016.

10 Spatial interpolation of undernutrition among late adolescent girls in Ethiopia

11 Ordinary Kriging method of interpolation analysis in EDHS 2000 showed that there was the

12 highest prediction of under-nutrition among late adolescent girls from unsampled areas in Tigray,

13 Afar, Eastern Amhara reigion, Southwestern and Southeastern Oromia, Somali, Southwestern

14 NNPR and parts of Beninshangul Gumuz regions, while the lowest rates of under-nutrition were

15 being predicted in Addis Ababa and its surrounding and some parts of Gambela region. From the

16 figure below the red color represented the highest predicted risk while, the green color indicated

17 the lowest rates of prediction (Fig. 5 A).

Similarly, EDHS 2005 revealed that the highest prediction of under-nutrition among late adolescent girls from unsampled enumeration areas were in Tigray, Northern Afar, Northeastern Amhara reigion. The lowest rates of under-nutrition during EDHS 2005 were observed in Addis Ababa, most parts of Oromia region and SNNPR (Fig.5 B).

22 Ordinary Kriging interpolation analysis of EDHS 2011 remarked that the highest prediction of 23 under-nutrition risk among late adolescent girls from unsampled cluster areas were observed in 24 Tigray, Afar, Northern and Eastern Amhara reigion whiele, the lowest rates of under-nutrition 25 were observed in Addis Ababa, Oromia and SNNPR region (Fig. 5 C).

26 Likewise, during EDHS 2016, the highest prediction of under-nutrition risk among late adolescent 27 girls from unsampled cluster areas were identified in Tigray, Afar, Eastern Amhara reigion and 
eastern Somali region, while, the lowest rates of under-nutrition were observed in Addis Ababa;

2 Oromia and SNNPR (Fig.5 D).

Figure 5: Ordinary kriging interpolation of under-nutrition among late adolescent girls in Ethiopia,

4 EDHS 2000-2016

5 Spatiotemporal scan statistical analysis of under-nutrition among late adolescent

6 girls in Ethiopia, EDHS 2000 to 2016 using SaTScan software 9.6

7 During EDHS 2000, a total of 153 significant clusters were identified in three clusters. The primary

8 significant big cluster of spatial windows encompassed Tigray, most parts of Afar and Northern

9 and Eastern Amhara regions. It was located at $13.883741 \mathrm{~N}, 39.162985 \mathrm{E}$ with $364.77 \mathrm{~km}$ radius.

Clusters in the primary windows were 1.31 times higher risk of undernutrition than those outside the window $(\mathrm{RR}=1.31, \mathrm{LLR}=13.46, \mathrm{P}$-value $<0.001)$. The primary small significant window included Harari and Northern Somali region which was centered at 9.506756 N, 42.621090 E with $51.27 \mathrm{~km}$ radius. It was 1.73 times higher risk of undernutrition than outside the windows $(\mathrm{RR}=1.73, \mathrm{LLR}=11.69 \mathrm{P}$-value $<0.01$. The second spatial window covered Southwest Oromia and Northern borders of SNNPR that was located at $8.245203 \mathrm{~N}, 37.785581 \mathrm{E}$ with $74.06 \mathrm{kms}$ radius. Clusters in the second widow was 1.59 times more risk than outside this window $R R=1.59$, $L L R=$ 10.03, P-Value < 0.05), respectively (Table 5 and Fig. 6 A). In EDHS 2005, a total of 36 significant the most likely clusters were identified that encompassed mainly Tigray region but also touched borders of Northern Amhara region. It was located at $14.108312 \mathrm{~N}, 38.288215 \mathrm{E}$ with $134.64 \mathrm{~km}$ radius. Clusters in primary windows were 1.79 times more risk than those outside the window $(\mathrm{RR}=1.79, \mathrm{LLR}=13.02$, P-value<0.001) (Table 5 and Fig. 6 B). During EDHS 2011, a total of 129 significant clusters were identified in the primary cluster. The significant cluster of spatial windows encompassed mainly Eastern and Southern Tigray region Afar, Eastern and Northern Amhara and parts of Northern Somali region. It was located at 12.635948 N, 40.297925 E with $308.64 \mathrm{~km}$ radius. Clusters in primary windows were 1.71 times higher risk than those outside the window ( $\mathrm{RR}=1.71, \mathrm{LLR}=67.07, \mathrm{P}$-value<0.001) (Table 5 and Fig. $6 \mathrm{C})$. During EDHS 2016 a total of 208 significant clusters were identified in three clusters. The most likely significant cluster of spatial windows encompassed central, Eastern and Southern Tigray, Afar and Eastern Amhara 
1 with $322.17 \mathrm{~km}$ radius. Clusters in the primary window were 1.77 times more risk than those

2 outside the window $(\mathrm{RR}=1.77, \mathrm{LLR}=54.45, \mathrm{P}$-value $<0.001)$. The second significant window

3 included mainly Gambela region but also borders of Western Oromia region. The third spatial

4 window encompassed most of parts Somali region and around a boundary of Oromia region. The

5 second and the third windows were located at $8.309769 \mathrm{~N}, 33.805118 \mathrm{E} / 105.98 \mathrm{~km}$ radius and

$6 \quad 5.589269 \mathrm{~N}, 44.175032 \mathrm{E}$ with $355.80 \mathrm{~km}$ radius. Clusters in second and third widows were 1.78

7 and 1.66 more higher risk of undernutrition than outside these windows $(R R=1.78, L L R=14.92$,

$8 \mathrm{P}$-value $<0.001$ and $\mathrm{RR}=1.66, \mathrm{LLR}=11.19, \mathrm{P}-$ Value $<0.01)$, respectively (Table 5 and fig6).

9 Figure 6: Cluster analysis of under-nutrition among late adolescent girls in Ethiopia, 2000, 2005, 102011 and 2016.

\section{Multilevel analysis}

\section{Model comparison and cluster variation}

13 Model comparison was done using AIC. Model IV or full model with the smallest value of AIC 14 (3511.477) was taken as the best fitted model (Table -6).

15 Table 6: Model comparison between DIC, AIC and BIC

\begin{tabular}{ll}
\hline Model & AIC \\
\hline I & 3721.823 \\
II & 3561.566 \\
III & 3650.787 \\
IV & $3511.477^{*}$ \\
\hline
\end{tabular}

*Best fitted model: AIC is the best fitted model (the smallest one)

17 The ICC value for null model was $15.53 \%$ which informed us to choose GLMM over the basic 18 model. (Table -7). The null model showed that under-nutrition among late adolescent girls was not 19 random across the communities $\left(\mathrm{VA}^{2}=0.60 ; \mathrm{P}<0.001\right)$.

20 Intercept only model revealed that $15.53 \%$ of variation in the odds of late adolescent girls' under21 nutrition could be attributed to community level factors based on the output of ICC value. The full 22 model after adjusting the individual and community level factors revealed that the variation in 
1 under-nutrition across the communities is statistically significant. About $11.6 \%$ of the odds of

2 under-nutrition variation across communities was observed in the full model (Table-7).

3 The MOR also showed that under-nutrition among late adolescent girls was attributed to

4 community level factors. The MOR for under-nutrition was 1.78 in the intercept only model; this

5 indicated that there is variation between communities (clustering). The MOR value was decreased

6 to 1.48 in model IV, when we added all variables that indicated the community level variations of

7 under-nutrition (Table-7).

8 The total variation attributed to individual and cluster level factors at each model was measured

9 by the proportional change in variance (PCV) which was computed as $1.87 \%, 39.76 \%$, and $31.67 \%$

10 in model II, model III and model IV respectively. This showed that there is clustering within

11 communities that informed us to use multilevel analysis (Table-7).

12 Table 7: The measure of variation for under-nutrition among late adolescent girls at cluster level

13 by multilevel binary logistic regression analysis

\begin{tabular}{|c|c|c|c|c|c|c|c|c|}
\hline $\begin{array}{l}\text { Measure of } \\
\text { variation }\end{array}$ & $\begin{array}{l}\text { Model - I } \\
\text { a }\end{array}$ & $\begin{array}{l}\mathrm{p}- \\
\text { value }\end{array}$ & Model-II $^{\mathrm{b}}$ & $\begin{array}{l}\mathrm{P}- \\
\text { value }\end{array}$ & $\begin{array}{l}\text { Model-III } \\
\mathrm{c}\end{array}$ & $\begin{array}{l}\mathrm{P}- \\
\text { value }\end{array}$ & $\begin{array}{l}\text { Model- IV } \\
\mathrm{d}\end{array}$ & $\begin{array}{l}\mathrm{P}- \\
\text { value }\end{array}$ \\
\hline Community & 0.6047 & $<0.00$ & 0.5934 & $<0.001$ & 0.3643 & $<0.001$ & 0.4132 & $<0.001$ \\
\hline $\begin{array}{l}\text { level Variance } \\
\text { (SE) }\end{array}$ & $(0.1057)$ & 1 & $(0.1259)$ & & $(0.079)$ & & $(0.0945)$ & \\
\hline ICC \% & 15.53 & & 5.39 & & 9.97 & & 11.16 & \\
\hline PCV \% & Ref. & & 1.87 & & 39.76 & & 31.67 & \\
\hline MOR & 1.78 & & 1.76 & & 1.41 & & 1.48 & \\
\hline \multicolumn{9}{|c|}{ Model fit statistics for best fitted model } \\
\hline AIC & 3721.823 & & 3561.566 & & 3650.787 & & $3511.477 *$ & \\
\hline
\end{tabular}

$\mathrm{SE}=$ standard error; $\mathrm{ICC}=$ intraclass correlation; $\mathrm{PCV}=$ Proportional Change in Variance, expresses the change in the cluster level variance between the null model and the individual level model, and between the individual level model and the model further including the community level covariates; MOR=Median Odds Ratio; DIC=Davian's information criteria Model I ${ }^{\mathrm{a}}-$ Empty model without any explanatory variable.

Model III ${ }^{c}$ - Adjusted for community-level factors

Model IV ${ }^{\mathrm{d}}$ - Full model adjusted for both individual and community-level factors 
1 Determinants of under-nutrition among late adolescent girls in Ethiopia

2 Individual and community level factors were selected using enter methods at 0.2 significance level

3 in bi-variable analysis.

4 The results of multilevel logistic regression models for individual and community level factors are

5 showed in Table below. In the final full model, that means all individual and community level

6 determinants are combined, factors such as late adolescent girls' age, educational status, marital

7 status and occupational status, age of household head, time to get drinking water, listening to radio

8 type of toilet facility, region and place of residence were significantly associated with late

9 adolescent girls' under-nutrition (Table -8).

\section{Individual level factors}

11 The odd of being under-nourished among late adolescent girls in the age group of 18 -19 years 12 were $57 \%$ (AOR $=0.43$; $95 \%$ CI: $(0.35-0.53)$ lower than those $15-17$ years old. Late adolescent 13 girls with higher educational status were 4.50 times (AOR=4.50; 95\% CI: $(2.33-8.69)$ more likely 14 to be under-nourished than those with no educational status. Late adolescents with divorced marital status were $46 \%$ (AOR=0.54, 95\% CI: $(0.30-0.97)$ less likely for undernutrition compared with never married. The odd of being under-nourished among late adolescent girls with occupation of sales were $40 \%$ ( $\mathrm{AOR}=0.60 ; 95 \% \mathrm{CI}: 0.43-0.84$ ) lower than not working adolescents. Late adolescent females with household head in the age group of 35-44 were 1.79 times ( $\mathrm{AOR}=179$, 95\% CI: (1.13 - 2.83) than 15-24 years. The odds of undernutrition were 1. 77 times (AOR=1.77; 95\% CI: (1.24 - 2.53) higher than those participants with un-improved latrine type. Participants with frequency of listening to radio at least once a week were $28 \%$ (AOR $=0.72 ; 95 \%$ CI: $(0.56$ -0.93 ) less likely to be under-nourished than those without listening radio (Table -8).

\section{Community level factors}

The odds of late adolescent girls' under-nutrition in Amhara, 47\% (AOR= 0.53; 95\% CI: $(0.35$ Tigray region respectively. $(\mathrm{AOR}=2.35 ; 95 \% \mathrm{CI}: 1.41-3.92)$ than urban residents (Table -8$)$. 


\section{Discussion}

2 The current study showed that the prevalence of under-nutrition among late adolescent girls was

3 decreased through over time on the past 15 years in Ethiopia. It had decreased from 36.79\%, 95\%

4 CI: (34.11-39.54) in EDHS 2000 to 28.22\%, 95\% CI: (26.02-30.52\%) in EDHS 2016. This could

5 be due to the fact that the government gives attention for improvement of agricultural production

6 and productive safety net programs in Ethiopia. However, there was regional variation of under-

7 nutrition. Four regions namely, Tigray, Afar, Amhara and Somali had higher prevalence (greater

8 than national average) during each survey period, while Addis Ababa and Oromia regions were in

9 the lower prevalence. This might be due to the fact that decreased amount of rainfall distribution

10 and recurrent drought attack in Northern and Eastern parts of the country. Under-nutrition was

11 spatially clustered nationally during each survey. The spatial distribution of under-nutrition during

12 each survey period was mainly in Northern, Eastern and some Western parts of the country

13 including Tigray, Afar, Eastern Amhara, Harari, Somali and Gambela regions. These variations

14 might be because of recurrent attack of drought, climate changes and rain fall distributions, land

15 degradation and soil erosion for crop production in above regions of Ethiopia in different time

16 periods. In this study, the local spatial statistics showed that spatial variation of under-nutrition in

17 different parts of Ethiopia. In 2000 survey, statistically significant hotspot areas of late adolescent girls' under-nutrition were observed in regions of Northern and Eastern Tigray, Afar, Eastern Amhara, Southwest \& Southeast Oromia, Somali and Northern and Western SNNPR, where as in 2005 survey, the hot spot areas of under-nutrition were restricted in Northern and North Eastern parts of the country. This might be related with seasonal attack of drought and decreased rainfall distributions which makes difficulties for crop production. Likewise, during 2011, the hot spot areas of under-nutrition were observed mainly in three regions (Tigray, Western and Eastern Afar and Northern and Eastern Amhara regions), while in 2016 survey, statistically significant hot spot areas were observed in the regions of Tigray, Northern eastern Amhara, Afar, Eastern Somali region, and Gambela region. This might be attributed with recurrent attack of drought in Northern and Eastern parts of the country. Agriculture is mainly affected by climate changes in Ethiopia (27). During 2015, El Niño drought which was one of the strongest droughts in Ethiopian that caused more than 27 million people for became food insecure that is one of the determinants of undermatron (4). 
This study also revealed that the highest prediction of under-nutrition prevalence was observed in

2 different regions in different time periods. In 2000 survey, the highest prediction of undernutrition

3 prevalence among late adolescent girls from unsampled areas were observed in regions of Tigray,

4 Afar, Eastern Amhara reigion, Southeast and Southwest Oromia, Somali region, Western NNPR

5 and parts of Beninshangul Gumuz regions. Likewise, EDHS 2005 revealed that the highest

6 prediction of under-nutrition from unsampled enumeration areas were seen in Tigray, Northern

7 Afar, Northern and Eastern Amhara reigion. Similarly, EDHS 2011 showed that the highest

8 prediction was restricted in three regions ( Tigray, Afar and Northern \& Eastern Amhara). During

9 EDHS 2016, the highest prediction of under-nutrition among late adolescent girls from unsampled

10 enumeration areas were observed in Tigray, Afar, Eastern Amhara reigion, Southern Somali

11 region and Gambell region.

12 This study showed that a number of statistically significant clusters with high and low rates that

13 were mainly observed in Northern and Eastern parts of Ethiopia during survey periods. In 2000

14 survey, there were a total of 153 statistically significant SaTScan clusters detected for undernutrition with high prevalence that encompassed Tigray, Afar and Amhara regions, whereas during 2005 survey, a total of 36 significant clusters were identified in the primary cluster that encompassed mainly Tigray region. Likewise, during EDHS 2011 a total of 129 significant clusters with high prevalence of under-nutrition were identified in the primary cluster that encompassed mainly Eastern and Southern Tigray region, Afar, Eastern Amhara and Northern Somali region. during EDHS 2016 a total of 208 significant clusters were identified in three clusters in which the primary significant cluster of spatial windows encompassed central, Eastern and Southern Tigray, Afar and Eastern Amhara region and Northern Somali region. The second significant window included Gambela and Western Oromia regions and the third spatial window encompassed most of parts Somali region and around a boundary of Oromia region. These high rates of undernutrition with significant clusters in different regions may be attributed to because of 2015 El Nino drought that affected food securities in above regions because of significant rainfall decrement and many livestock deaths in Afar, Somali and Oromia pastoralists $(4,28)$.

The current study identified that the regional variation of under nutrition among late adolescent girls was attributed to both individual and community level factors. In the final full model, individual and community-level factors accounted for about $31.67 \%$ of the variations for under- 
nutrition among late adolescent girls. Multilevel analysis of this study showed that different

2 individual and community level factors influenced under-nutrition among late adolescent girls in

3 Ethiopia.

4 In individual level factors, the age groups of late adolescent girls were significantly associated

5 with under-nutrition: The odds of being under-nourished among late adolescent girls in the age group of $18-19$ years were 57\% less likely than those 15-17 years old. This may be because of synergistic effect of growth velocity during puberty when peak height velocity occurs and endocrine factors are also essential for promoting normal adolescent growth and are sensitive to under-nutrition (12). This was supported by a study in Northwestern Ethiopia (29), the age groups of 15-17 years were 2 times more higher for being under-nourished than 18-19 years old adolescents. On the other hand, adolescent girls after 18 years may be engaged into marriage and may be better access to eating patterns in economically limited families. In contrast with above, study from India (30) showed late adolescents were less likely for undernutrition compared with 14 early adolescents.

The current study identified that late adolescent girls with higher educational status were 4.50 times more likely to be under-nourished compared with no educational status. This may be attributed to girls are more vulnerable for the influences of cultural and gender norms, which often discriminate against frequent feeding and when dietary intakes are suboptimal, anemia and micronutrient deficiencies are high among adolescent girls (12). On the other hand, during higher education, those adolescents may go to place far from their parents, so that they are limited to get timely feeding and food varieties due to economic barriers. A study stated that eating patterns and behaviors are influenced by peer pressure, food availability, food preferences and cost, personal and cultural beliefs (1). A study from low and middle-income countries also remarked that about $40 \%$ of adolescent girls reported skipping their breakfast (31).

This study identified that late adolescent girls with occupation of sales were $40 \%$ lower for being under-nourished than those with not working. This might be because of they have their own money who easily to purchase varieties of food items.

The current study stated that the odds of under-nutrition was 1.77 times higher than participants with un-improved latrine type. This may be attributed to poor sanitation could expose infestation 
of intestinal parasites that leads to illness, poor appetite and micronutrient deficiencies that leads

2 to undernutrition. This was consistent with SRMA study in Ethiopia (6).

3 Participants with frequency of listening to radio at least once a week were $28 \%$ less likely to be

4 under-nourished than those without listening radio. This could be because of better awareness and

5 information gain regarding importance of variety of food items and frequency of feeding patterns

6 among those with listening radio.

7 In the current study, community level factors were significantly associated with under-nutrition.

8 The odds of late adolescent girls' under-nutrition in the regions of Amhara, Oromia, Benishangul

9 Gumuz and SNNPR was 47\%, 66\%, 82\% and 77\% lower compared with Tigray region, respectively. This might be because of divergence to access of food security across regions in Ethiopia due to climate changes, rain fall distributions and soil degradation and erosion for crop production (4). This was supported with study done in Amhara region that showed adolescent girls living in food secured households were 35\% less likely to be undernourished than their counter parts (29). Additionally, SRMA study in Ethiopia showed that adolescent girls from food insecured households were 2.38 times more higher for being short stature than food secured families (6).

This study revealed that the odds of late adolescent girls' under-nutrition among rural residents was increased by $2.35 \%$ compared with urban residents. This was consistent with a recent SRMA study in Ethiopia that remarked being rural residence was 2.19 times more higher for being undernourished than urban residents (6). This might be attributed to cultural influences, lack of awareness about importance of variety of foods and due to food insecurity in rural areas because of recurrent drought, climate changes and land degradation and soil erosion (4). It was supported by a research in North western Amhara region (8) which stated that the odds of stunting was $45 \%$ higher among adolescents of rural areas with food insecured households. On the other hand, systematic review study done among adolescent girls in low- and middle-income countries that showed the mean energy intake was lower in rural settings compared to urban settings (31).

27 Strength and limitation of the study: Since we used four consecutive large data sets of EDHS, the study was nationally representative. Spatiotemporal analysis was used to explore hotspot and high-rate significant cluster analysis that would be important to design interventions at community 


\section{Conclusion:} in each survey.

\section{Declarations:}

better estimate of level of association. Since it was secondary data analysis, we didn't find some important variables, such as food security, variety of foods and clinical related variables and variables like husband educational status and occupational status were not consistently collected

The current study found that under-nutrition among late adolescent girls was clustered across regions in Ethiopia in each survey. The spatio-temporal patterns of this study showed that there was high spatial dependency across regions. The spatial scan statistics revealed that the significant clusters with high prevalence of under-nutrition encompassed Northern, Eastern and also Western parts of Ethiopia recently. Age groups, educational status and occupational status of adolescents, un-improved latrine type, frequency of listening to radio, head of household head, being rural residents and region, were significantly associated with undernutrition. Therefore, the government of Ethiopia and stakeholders should take responsibilities in these areas to intervene undernutrition early. Awareness creation via mass-media and health education regarding on importance of using an improved latrine is mandatory.

\section{List of abbreviations:}

AIC- Akaike's Information Criterion; AOR- Adjusted Odds Ratio; ArcGIS- Geographic Information System; BMI- Body Mass Index; DALIS- Disability Adjusted Life Years; DHSDemographic Health Survey; EAs - Enumeration Areas; EDHS- Ethiopian Demographic and Health Survey; GLMM- Generalized Linear Mixed Model; HH- Household; ICC- Intra-class Correlation Coefficient; IQR- Inter Quartile range; LLR- Log Likelihood Ratio; LBW- Low Birth Weight; MCH- Maternal and Child Health; MOR- Median Odds Ratio; PCV- Proportional Change in Variance; SD- Standard Deviation; SNNPR- South Nations Nationalities and Peoples Representatives; TB- Tuberculosis

\section{Ethical approval and consent to participate:}

Permission from DHS program data base was obtained after being registered and submitted our research question. Ethical approval was obtained from University of Gondar Research Ethics Review committee. Data was handled with confidentially during all phases of research activities. 
1 Consent to participant for this particular study is not applicable since the study was a secondary

2 data analysis of publicly available survey data from MEASURE DHS program.

3 Acknowledgments:

4 Our deepest thanks go to DHS Programs which granted us the permission to use DHS data. We

5 would also like to thank the University of Gondar College of Medicine and Health Sciences,

6 Institute of Public Health and Department of Epidemiology and Biostatistics staffs for their

7 support.

\section{Authors contribution:}

9 NMD: conceived and designed the study, data extraction, writing the original draft and review, conducted analysis and interpretation, report writing and critically reviewed the manuscript and

11 did final editing.

12 KAG: conceptualised and designed the study, validation and visualization, critically reviewed the 13 manuscript

14 AGM: conceptualised and designed the study, assisted in the data analysis and interpretation, 15 validation and Visualization and critically reviewed the manuscript. All of the authors read and 16 approved the final version of the manuscript

17 Funding: The authors have not declared any funding for this study.

18 Competing interests: The authors declare that they have no competing interests.

19 Availability of data and materials:

20 The availability of data for this particular study was from the DHS program datasets using the

21 website www.measuredhs.com. after we had sent the research question

22 Consent for publication:

23 It is not applicable for this study since the study was secondary data analysis of national survey

24 collected by central statistics agency.

25 Contributors information:

26 Nebiyu Mekonnen Derseh: nebiyumek12@gmail.com,

27 Kassahun Alemu Gelaye: kassalemu@gmail.com 
Atalay Goshu Muluneh: goshuatalay12@gmail.com

2

\section{References:}

1. Das JK, Salam RA, Thornburg KL, Prentice AM, Campisi S, Lassi ZS, et al. Nutrition in adolescents: physiology, metabolism, and nutritional needs. Annals of the New York Academy of Sciences. 2017;1393(1):21-33.

2. USAID. Adolescent nutrition 2000-2017: DHS data on adolescents age 15-19. ICF Rockville, Maryland, USA; June 2018.

3. WHO. Guideline: Implementing effective actions for improving adolescent nutrition. In: Organization WH, editor. Geneva:2018.

4. Mohamed AA. Food Security Situation in Ethiopia: A Review Study. International Journal of Health Economics and Policy. 2017;2(3):86-96.

5. Agency CS, [Ethiopia], and, International I. Ethiopia Demographic and Health Survey 2011. Addis Ababa, Ethiopia and Calverton, Maryland, USA: . ICF International Calverton, Maryland, USA; March 2012.

6. Berhe K, Kidanemariam A, Gebremariam G, Gebremariam A. Prevalence and associated factors of adolescent undernutrition in Ethiopia: a systematic review and meta-analysis. BMC Nutrition. 2019;5(1):49.

7. [Ethiopia] CSAC, and, ICF. Ethiopia Demographic and Health Survey 2016. Addis Ababa, Ethiopia, and Rockville, Maryland, USA: CSA and ICF. Addis Ababa, Ethiopia; 2016.

8. Amare Tariku AKB, Hunegnaw MT, ZA, Abebe SM, Fekadud A, KFM, et al. Stunting and Its Determinants among Adolescent Girls: Findings from the Nutrition Surveillance Project, Northwest Ethiopia. Ecology of Food and Nutrition. 04 Jul 2019.

9. UNAIDS. Multi-Sectoral Nutrition Strategy Global Learning and Evidence Exchange East and Southern Africa Regional Meeting. Nutrition for adolescent girls and women What do we know about what works? Kavita Sethuraman, FANTA III, FHI 360: FANTA; 2018S.

10. Cross IFoR, and Red Crescent Societies. Nutrition guidelines. In: Cross IFoR, and Red Crescent Societies, editors. InGeneva, 20132013. p. 72.

11. Farah AM, Endris BS, Gebreyesus SH. Maternal undernutrition as proxy indicators of their offspring's undernutrition: evidence from 2011 Ethiopia demographic and health survey. BMC Nutrition. 2019;5(1). 
12. Parul Christian a bERSa, c. Adolescent Undernutrition: Global Burden, Physiology, and Nutritional Risks. Ann Nutr Metab 2018;72:316-28.

13. Ahmed F, Khan MR, Banu CP, Qazi MR, Akhtaruzzaman M. The coexistence of other micronutrient deficiencies in anaemic adolescent schoolgirls in rural Bangladesh. Eur J Clin Nutr. 2008;62(3):365-72.

14. Andiran N, Celik N, Akca H, Dogan G. Vitamin D deficiency in children and adolescents. Journal of clinical research in pediatric endocrinology. 2012;4(1):25-9.

15. Regasa RT, Haidar JA. Anemia and its determinant of in-school adolescent girls from rural Ethiopia: a school based cross-sectional study. BMC Womens Health. 2019;19(1):98.

16. Tariku A, Gonete KA, Bikes GA, Alemu K, Belew AK, Wassie MM, et al. Household food insecurity predisposes to undiversified diet in northwest Ethiopia: finding from the baseline survey of nutrition project, 2016. BMC Res Notes. 2019;12(1):54.

17. Roba KT*, and AM, T W. Nutritional Status and Its Associated Factors among School Adolescent Girls in Adama City, Central Ethiopia. J Nutr Food Sci. 2016;6:3.

18. Mahoo H, Raden My, Kinyangi J, Cramer L, eds. Climate Change Vulnerability and Risk Assessment of Agriculture and Food Security in Ethiopia Which Way Forward? CGIAR Research Program on Climate Change, Agriculture and Food Security (CCAFS). 2013:294.

19. Nations U, Division P, Affairs DoEaS. World Population Prospects :Total population (both sexes combined) by region, subregion and country, annually for Estimates, 1950 - 2020: ; 2019

20. International. I. Demographic and Health Survey Sampling and Household Listing Manual. In: MEASURE DHS, editor. Calverton, Maryland, U.S.A: ICF International; 2012.

21. [Ethiopia] CSA, and, Macro. O. Ethiopia Demographic and Health Survey 2000. Addis Ababa, Ethiopia and Calverton, Maryland, USA; 2001.

22. [Ethiopia] CSA, and, Macro O. Ethiopia Demographic and Health Survey 2005. . Addis Ababa, Ethiopia and Calverton, Maryland, USA; 2006.

23. Elsevier. -Encyclopedia of human nutrition-Elsevier Science (E) SECOND EDITION ed. <Benjamin Caballero LA, Andrew Prentice editor. Oxford, OX5 1GB, UK: Elsevier ACADEMIC PRESS; 2005. 2167 p.

24. W. J. - Generalized Linear Models and Extensions-Stata Press (2018).pdf >. Fourth ed. Hardin JMH, editor. 4905 Lakeway Drive, College Station, Texas 77845: Stata Press; 2018. 789 p. 
1 25. SAGE. The SAGE Handbook of Multilevel Modeling. Marc A. Scott, and JSS, Marx BD, 2 editors2013.

3 26. Merlo J, Chaix B, Ohlsson H, Beckman A, Johnell K, Hjerpe P, et al. A brief conceptual tutorial 4 of multilevel analysis in social epidemiology: using measures of clustering in multilevel 5 logistic regression to investigate contextual phenomena. Journal of epidemiology and 6 community health. 2006;60(4):290-7.

7 27. Gezie M, Tejada Moral M. Farmer's response to climate change and variability in Ethiopia: A 8 review. Cogent Food \& Agriculture. 2019;5(1):1613770.

9 28. FAO. ElNino-Response Plan. America; 2016.

10 29. Molla Mesele Wassie1*, Azeb Atnafu Gete1, Melkie Edris Yesuf1, Getu Degu Alene2, Belay3 11 A, and, et al. Predictors of nutritional status of Ethiopian adolescent girls: a community based 12 cross sectional study. BMC Nutrition. 2015;1:20.

13 30. Ahmad S, Shukla NK, Singh JV, Shukla R, Shukla M. Double burden of malnutrition among 14 school-going adolescent girls in North India: A cross-sectional study. Journal of family 15 medicine and primary care. 2018;7(6):1417-24.

16 31. Keats EC, Rappaport AI, Shah S, Oh C, Jain R, Bhutta ZA. The Dietary Intake and Practices 17 of Adolescent Girls in Low- and Middle-Income Countries: A Systematic Review. Nutrients. $18 \quad 2018 ; 10(12)$. 


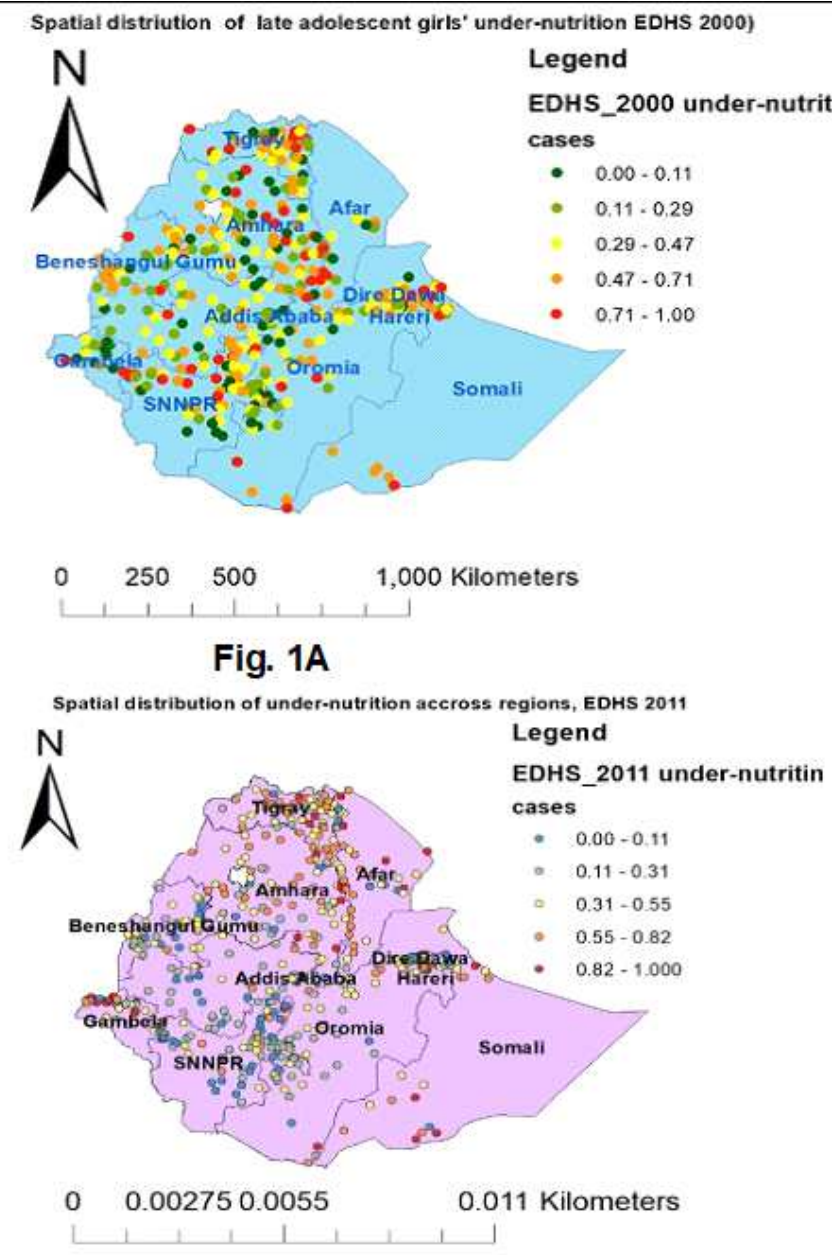

Fig. 1C
Spatial distributions of undder-nutrition amomog late adolescent girls, EDHS 2005

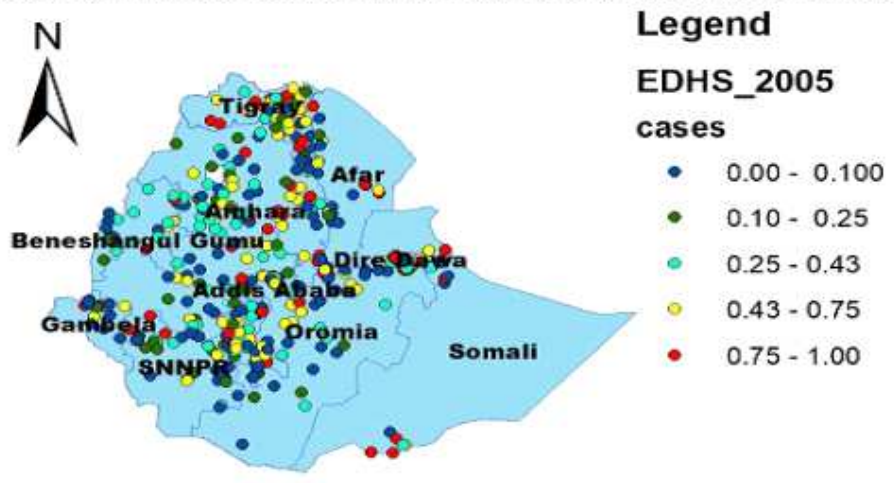

$0.002250 .0045 \quad 0.009$ Kilometers

Fig. 1B

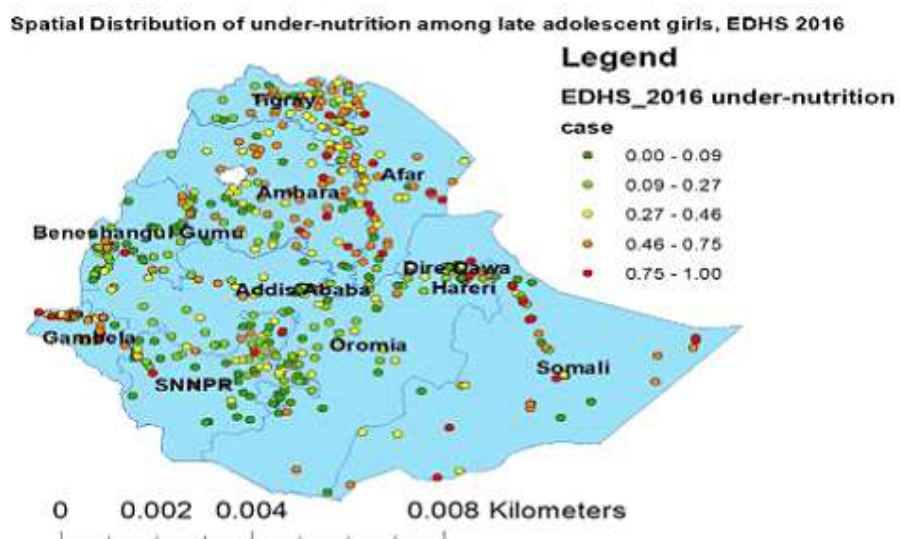

Fig. 1D

Source: Ethiopian CSA, 2013

\section{Figure 1}

Spatial distribution of late adolescent girls' under-nutrition in Ethiopia, EDHS 2000 to 2016.

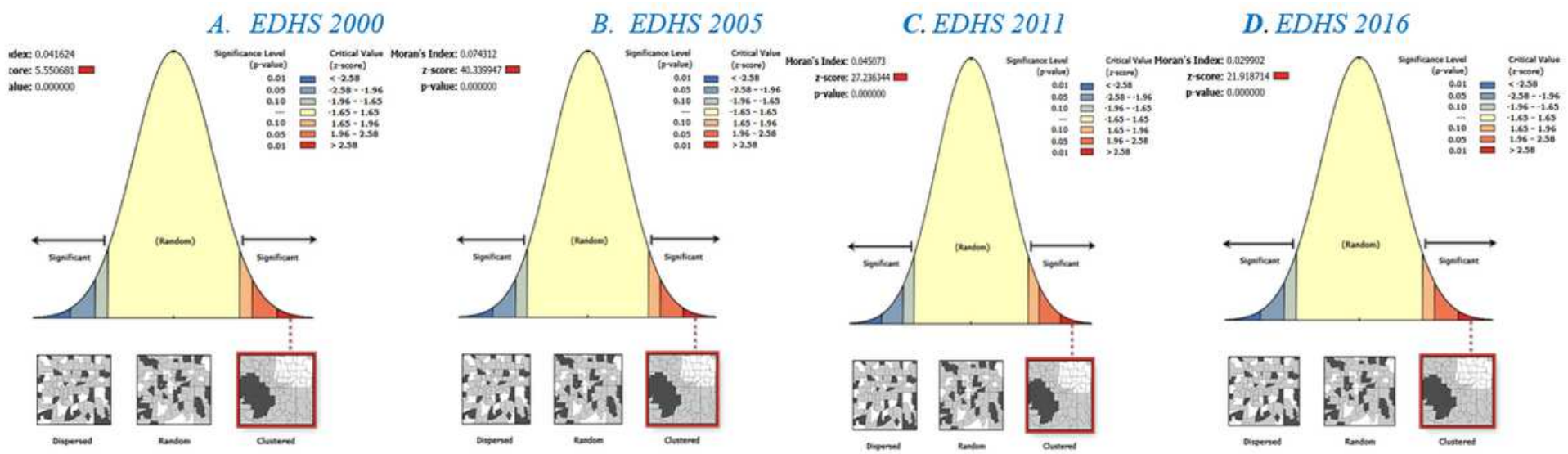

Figure 2 


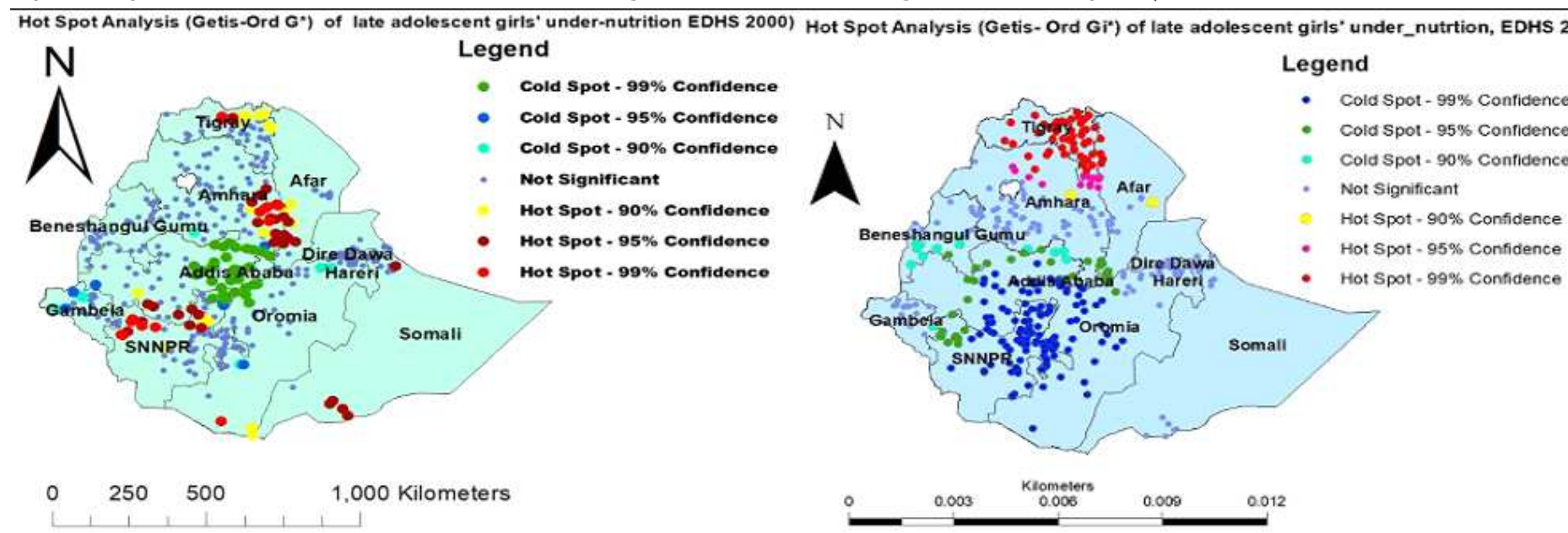

Fig. 3A

Hot spot Analysis (Getis-Ord $\mathrm{Gi}^{*}$ ) of under-nutrition among late adoleseent giris $\tilde{\Lambda}$ Legend

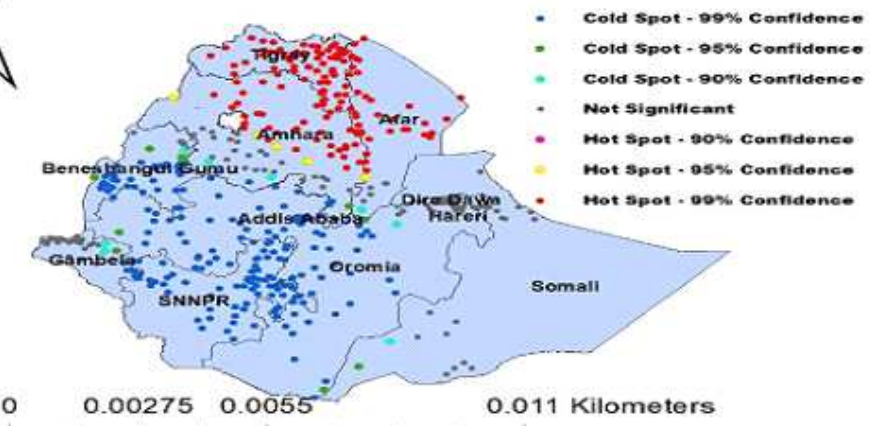

Fig. 3C

Fig. 3B

Hot Spot Anaysis of late adolescent girls' under-nutrition, EDHS 2016

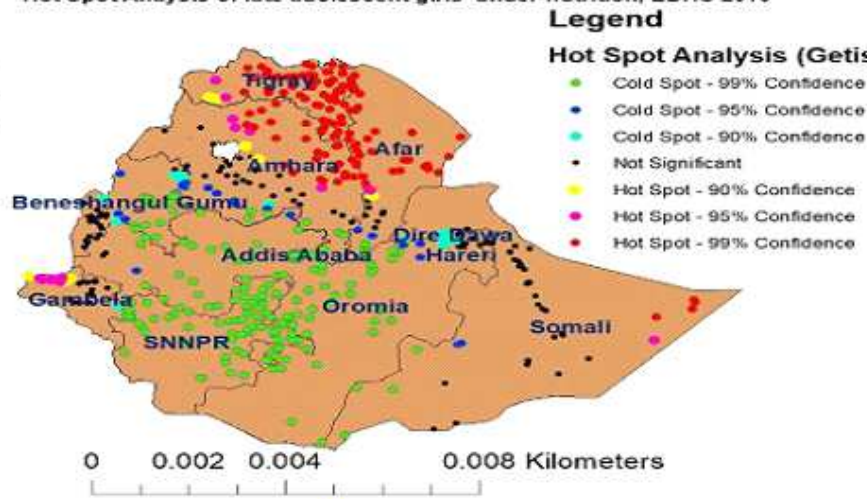

Fig. 3D

\section{Figure 3}

Hotspot analysis of under-nutrition among late adolescent girls in Ethiopia, 2000, 2005, 2011 and 2016 

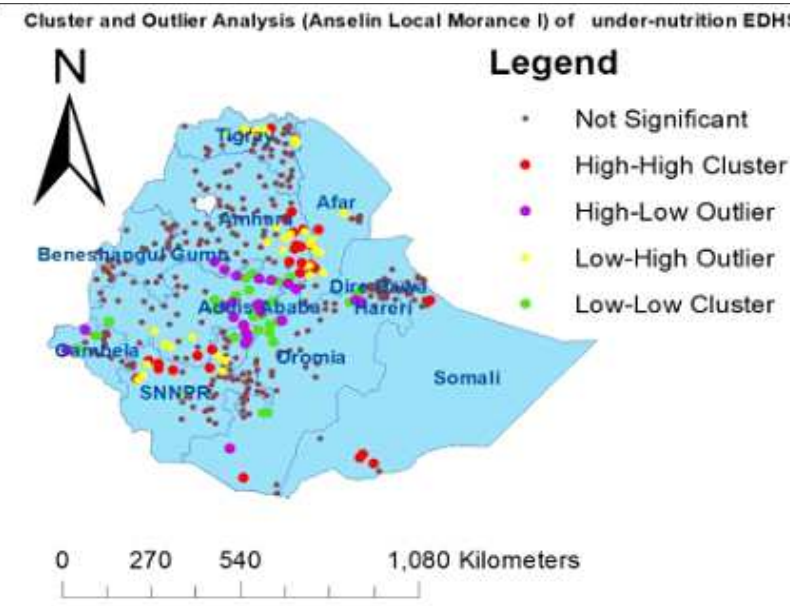

Fig 4A

Cluster and Outlier Analysi (Anselin Local Morans 1) of under-nutrition

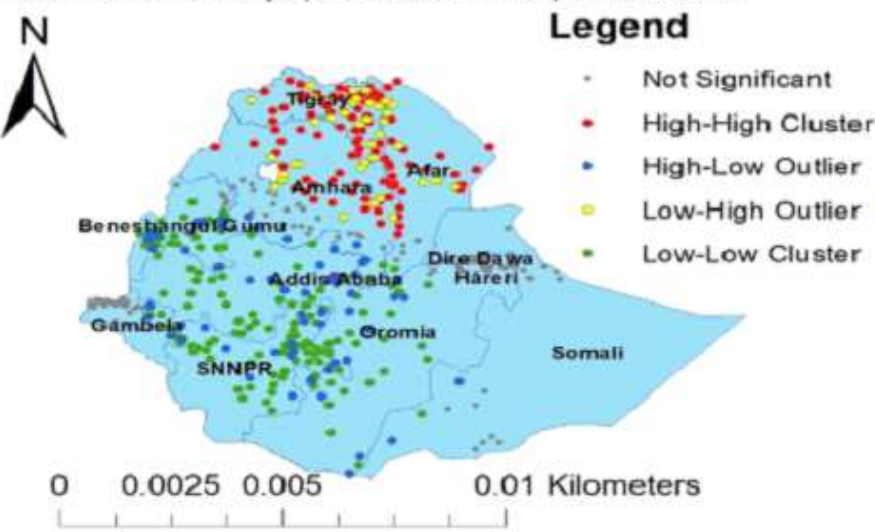

Fig. 4C

Source: Ethiopian CSA, 2013

\section{Legend}

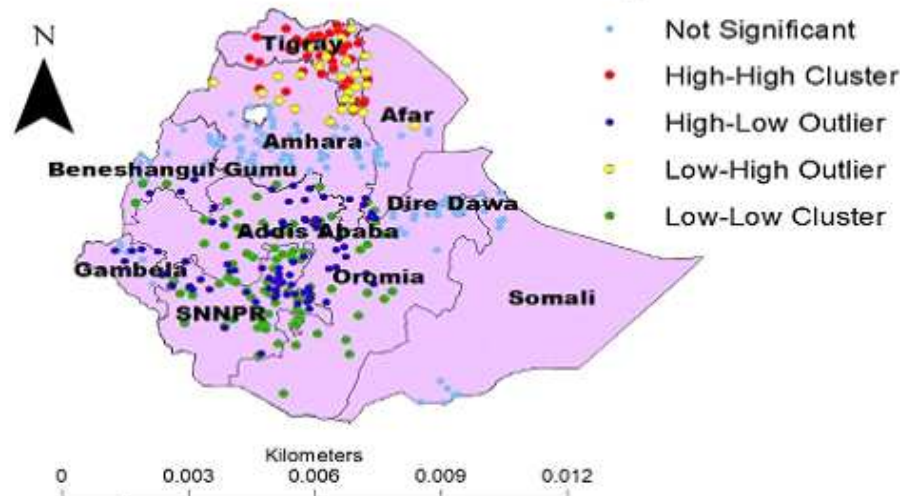

Fig 4B

Cluster and Outlier Anaysis (Anselin Local Moran's 1) of under-nutrition EDHS 2016

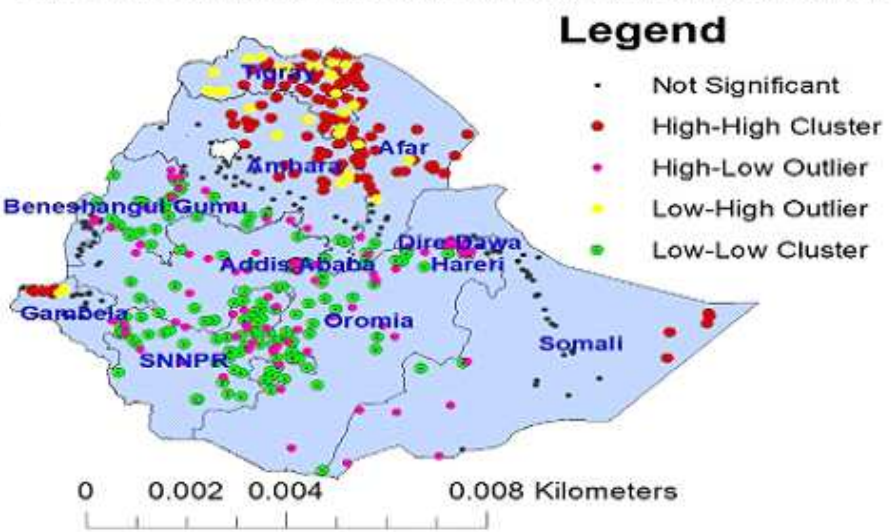

Fig. 4D

\section{Figure 4}

Cluster and Outlier Analysis of undernutrition among late adolescent girls in Ethiopia, EDHS 2000 to 2016. 


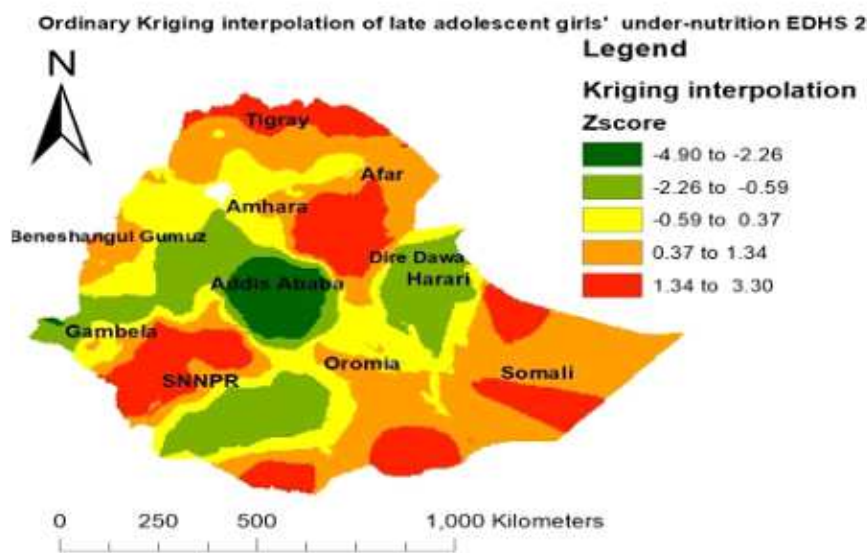

Fig. 5A

Ordinary Kriging interpolation of under-nutrition accross regions, EDHS 2011

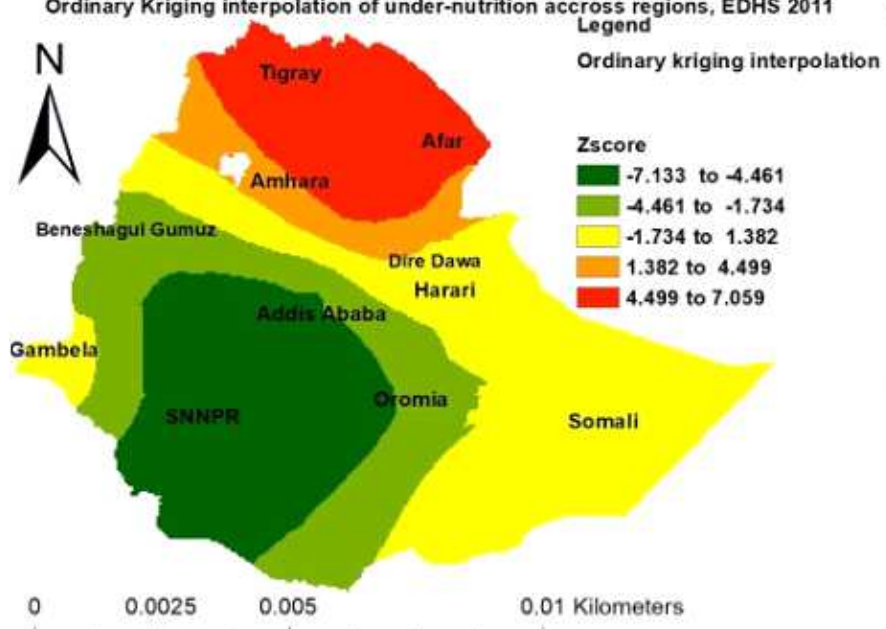

Fig. 5C
Ordinary Kriging interpolation of under-nutrtion among late adolescent girls, EDHS 2005

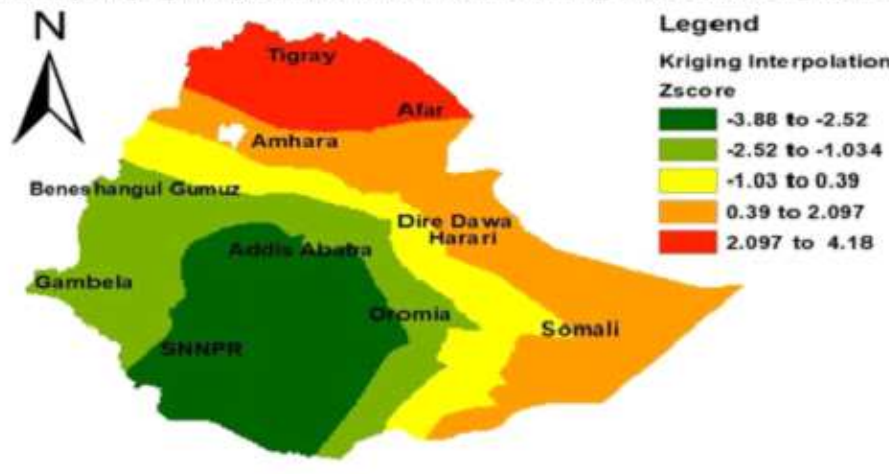

0 $0.002 \quad 0.004$ 0.008 0.012 0.016

Fig. 5B

Ordinary Kringing interpolation of under-nutrition EDHS 2016
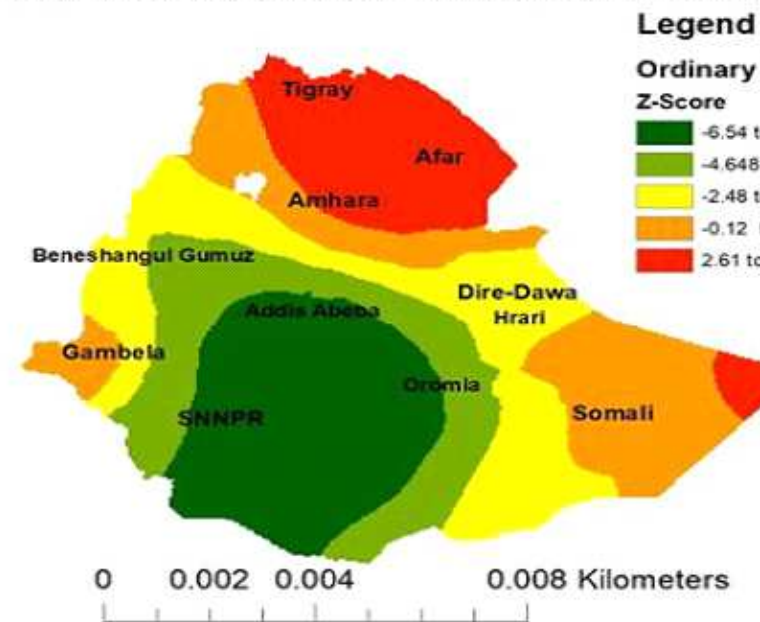

Legend

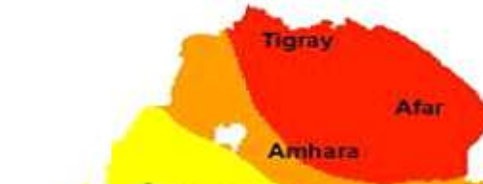

Beneshangut Gumuz

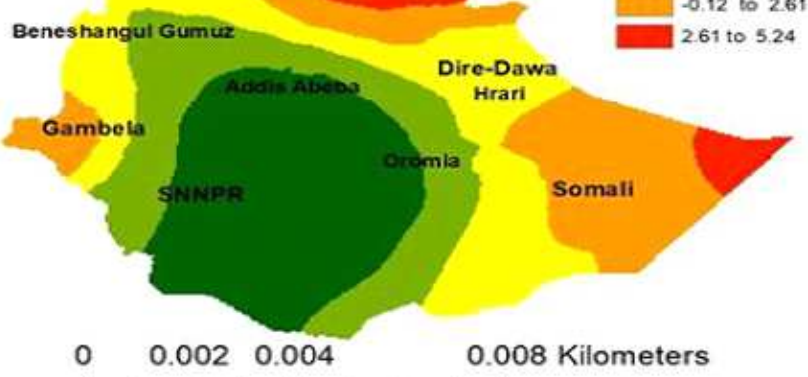
Z-Score 


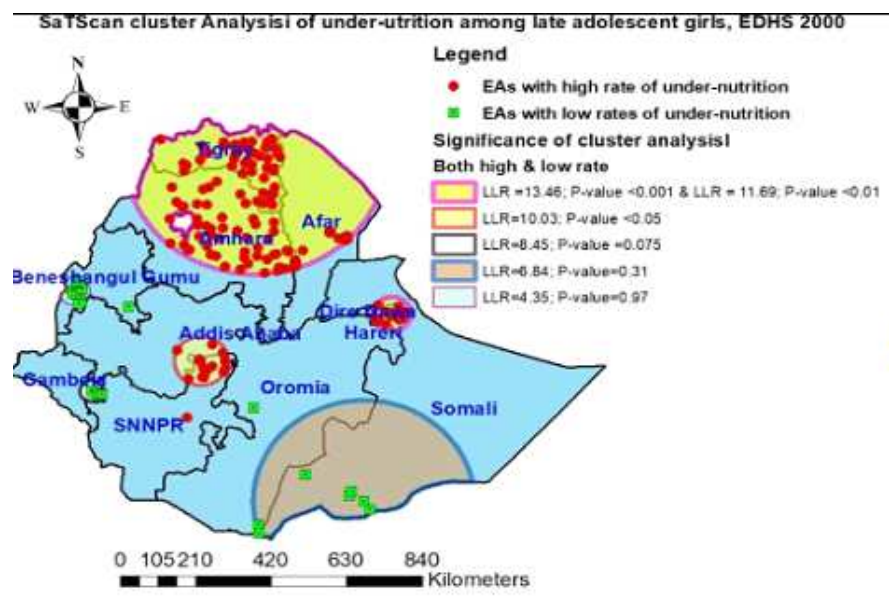

Fig. 6A

SaTScan Cluster anlysis of under-nutrition among late adolescent girls, EDHS 2011

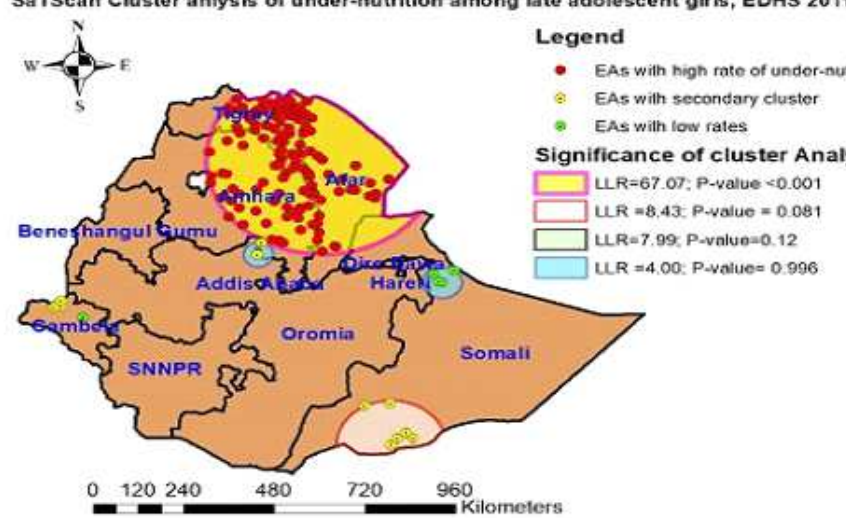

Fig. 6C

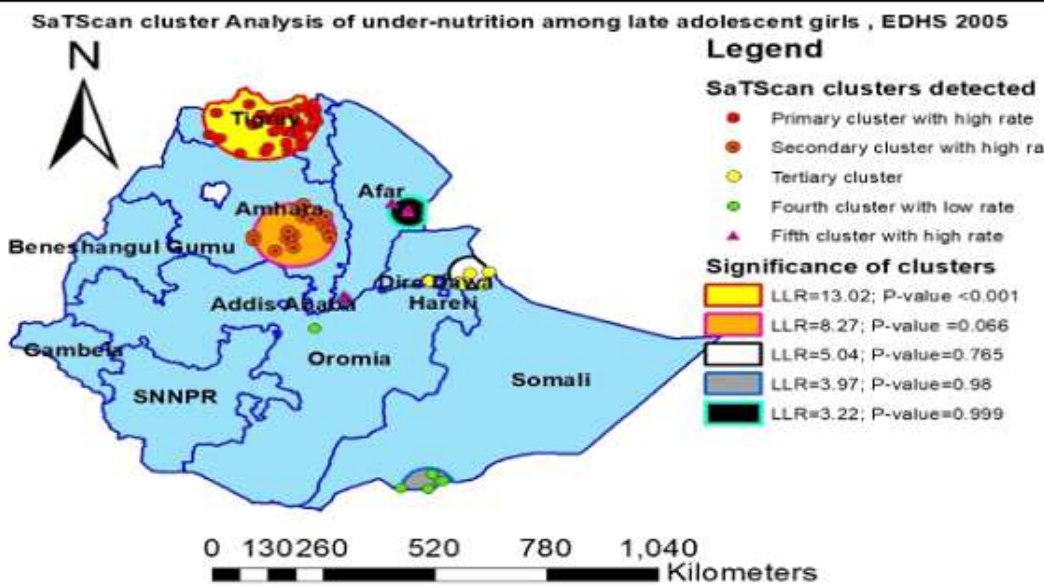

Fig. 6 B

SaTScan cluster Anaysis of under-nutrition among late adolescent girls, EDHS 2016

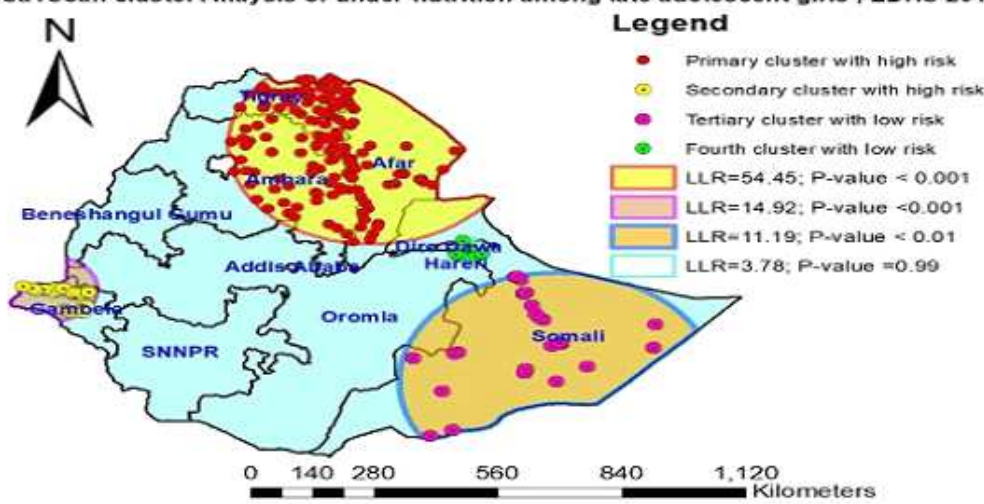

Fig. 6D

Source: Ethiopian CSA, 2013

Figure 6

Cluster analysis of under-nutrition among late adolescent girls in Ethiopia, 2000, 2005, 2011 and 2016.

\section{Supplementary Files}

This is a list of supplementary files associated with this preprint. Click to download.

- ListsofTable.docx 